\title{
Incidence, Microbiological Profile, and Impact of Preventive Measures on Central Line-associated Bloodstream Infection in Liver Care Intensive Care Unit
}

\author{
Arvind Khodare ${ }^{1}$, Pratibha Kale ${ }^{2}$, Girisha Pindi ${ }^{3}$, Lejo Joy ${ }^{4}$, Vikas Khillan ${ }^{5}$
}

\begin{abstract}
Aims and objectives: Central line-associated bloodstream infection (CLABSI) is among one of the preventable healthcare-associated infections (HAIs). The data for the CLABSI rate in liver care intensive care unit (LCICU) patients are scarce, so the present study was conducted to ascertain the CLABSI rate, the microbiological profile, and the impact of preventive measures for reduction of infection.

Materials and methods: This is a prospective observational study done on LCICU patients during the period of January 2017-December 2018. We followed up patients on the central venous catheter for the development of CLABSI as a part of routine surveillance of HAls. The impact of introduction and implementation of the CLABSI bundle to reduce the CLABSI rate was analyzed and the microbiological profile of infection was determined.

Results: During the study period, the total number of patients admitted in LCICU were 1,336 (648 in 2017 and 688 in 2018) and a total of 995 central lines were inserted for various indications. A total of 57 patients were meeting the CLABSI criteria among 7,324 central line catheter days of surveillance. In year 2017, rate of CLABSI was 11.78/1,000 catheter days and after implementation of the bundle in 2018 the rate reduced to $3.99 / 1,000$ catheter days. Gram-negative organisms (86\%) predominated with Pseudomonas aeruginosa being the most common pathogen (19.3\%). Out of 49 isolates of gram-negative bacilli (GNB), 40 (81.6\%) were multidrug resistant (MDR) and 9 (18.4\%) were pan-drug resistant.

Conclusion: We found significant reduction in the CLABSI rate after implementation of the bundle of care. Gram-negative bacilli were the most common pathogen in our study and antimicrobial resistance was very high, which suggest hospital environment as a source of infection. Clinical significance: Knowledge of the microbiological profile and the preventive strategy of CLABSI is essential for prevention and timely initiation of the most appropriate anti-infective therapy, if it happens.
\end{abstract}

Keywords: Bundle care, CLABSI, Healthcare-associated bloodstream infection, Infection control, Sepsis, Multidrug resistance.

Indian Journal of Critical Care Medicine (2020): 10.5005/jp-journals-10071-23325

\section{INTRODUCTION}

Central venous catheter (CVC) insertion is a common and often unavoidable invasive procedure in medical care of critically ill patients. The CVC is used for administering medications, fluids, blood products, total parenteral nutrition, and for hemodynamic monitoring. As these catheters have access to the bloodstream, it can result in significant complications, most importantly bloodstream infection (BSI). Central line-associated bloodstream infection (CLABSI) is among one of the preventable healthcareassociated infections (HAls), which results in increases in the duration of hospital stays and costs. ${ }^{1-3}$ For healthcare-associated BSIs, two closely related but distinct terms, "CLABSI" and "central line-related bloodstream infection (CRBSI)," are used. The term "CLABSI" is used for surveillance purposes while the term "CRBSI" is used often in the clinical context. ${ }^{4}$ In other words, CLABSI is a surrogate marker of CRBSI for surveillance purposes. Criteria used for the diagnosis of CLABSI are different and simpler than CRBSI to make the surveillance easy, objective, and universal. Central line-related bloodstream infection is diagnosed by demonstrating quantitative differences in bacterial load in blood cultures obtained from peripheral veins vs CVC, while the CLABSI has been defined by the National Healthcare Safety Network (NHSN) as a laboratoryconfirmed BSI where an eligible BSI organism is identified and an eligible central line is present on the laboratory-confirmed bloodstream infection-date of event (LCBI-DOE) or the day before. ${ }^{5}$
${ }^{1-5}$ Department of Microbiology, Institute of Liver and Biliary Sciences, New Delhi, India

Corresponding Author: Vikas Khillan, Department of Microbiology, Institute of Liver and Biliary Sciences, New Delhi, India, Phone: +91 9810686970, e-mail: vkhillanilbs@gmail.com

How to cite this article: Khodare A, Kale P, Pindi G, Joy L, Khillan V. Incidence, Microbiological Profile, and Impact of Preventive Measures on Central Line-associated Bloodstream Infection in Liver Care Intensive Care Unit. Indian J Crit Care Med 2020;24(1):17-22.

Source of support: Nil

Conflict of interest: None

Strategies most often used for prevention of healthcare-associated BSIs are surveillance and the bundle care approach. ${ }^{3}$ The Institute for Healthcare Improvement (IHI) describes bundles as "groupings of best practices with respect to a disease process that individually improve care, but when applied together result in substantially greater improvement." ${ }^{2}$ The condition of patients with BSIs in intensive care units (ICUs) has improved after the implementation of a central line insertion and maintenance bundle. ${ }^{1}$ Incidence of infection is more in developing countries as compared to developed countries. ${ }^{6}$ The high rates of CLABSI in ICUs has been attributed to poor compliance with the infection-control practices. In the ICU, the central line might be needed for extended periods of time especially in chronically ill patients, being manipulated several

(0) The Author(s). 2020 Open Access This article is distributed under the terms of the Creative Commons Attribution 4.0 International License (https://creativecommons. org/licenses/by-nc/4.0/), which permits unrestricted use, distribution, and non-commercial reproduction in any medium, provided you give appropriate credit to the original author(s) and the source, provide a link to the Creative Commons license, and indicate if changes were made. The Creative Commons Public Domain Dedication waiver (http://creativecommons.org/publicdomain/zero/1.0/) applies to the data made available in this article, unless otherwise stated. 
times, and leading to colonization with hospital flora. Moreover, some catheters may be inserted in urgent situations, during which optimal aseptic precautions may be compromised. ${ }^{2}$ Healthcareassociated infection surveillance is central to infection prevention and control efforts. Infection surveillance data is used to measure success of infection prevention and control programs, and to identify areas for improvement.

There is abundance of literature on the rate of CLABSI in general critical care units; however, data in ICU for liver patients is still scarce. So, this prospective surveillance study was conducted in the liver care intensive care unit (LCICU) of a tertiary liver care center on all patients with CVC to ascertain the incidence of CLABSI and to identify profile of the infecting microorganisms and the impact of implemented preventive measures to lower the CLABSI rate.

\section{Materials and Methods}

This prospective observational study was conducted in a 24-bedded LCICU of a tertiary liver care center in North India. All patients of age more than 18 years were admitted to LCICU during January 2017 to December 2018 and required the insertion of at least one nontunneled temporary CVC for any medical indication.

Surveillance for HAls was conducted daily by the infectioncontrol department across all ICU patients. We collected data prospectively using a $\mathrm{HAl}$ surveillance form as a routine protocol of infection-control activities. These included numerator data (patient demographic, clinical, CVC information, and diagnosis of a CLABSI event) and denominator data (the daily number of patients with or without (VC).

The definition of CLABSI used here was based on U.S. Centers for Disease Control and Prevention's National Healthcare Safety Network surveillance definitions. ${ }^{5}$ Central line-associated bloodstream infection is defined as a laboratory-confirmed BSI that is not secondary to an infection at another body site in patients who have at least one central line in place for more than 2 calendar days at the time of, or a day before, the onset of the event. ${ }^{5}$

A catheter day is defined as a single day on which a patient had one or more catheters.

The CLABSI rate is defined as a number of CLABSI per 1,000 CVC days or number of CLABSI per 1,000 ICU patient days.

The central line utilization ratio was calculated by using formula: number of central line days/number of patient days.

Blood culture was performed using BacT/ALERT Microbial Detection Systems (bioMérieux Inc., Durham, NC). Identification and antimicrobial susceptibility testing was done by the Vitek 2 (bioMérieux Inc., Durham, NC) system.

\section{INTERVENTION}

In December 2017 and January 2018, CVC insertion and maintenance bundle were applied for the prevention of CLABSI and was monitored using the checklist. The interventions applied were approved by the infection control committee of institute.

The CVC insertion bundle includes: (1) hand hygiene before catheter insertion, (2) optimal catheter site selection with avoidance of the femoral vein for central venous access in adult patients, (3) maximal barrier precautions upon insertion, and (4) chlorhexidine skin preparation.

The CVC maintenance bundle includes: (1) daily review of CVC need, (2) hand hygiene performed before and after all line maintenance/access procedures, (3) alcohol used to disinfect the catheter hub before each access, (4) insertion site inspection to see soiling of dressing and signs of inflammation, and (5) change the dressing using the aseptic technique.

Over the course of the study, the rate of CLABSI per 1,000 catheter days was monitored as the primary outcome. The aim of the interventions was to minimize the rate of CLABSI, with a goal of zero.

The statistical analysis was done using software SPSS Statistics Version 20. The CLABSI rate was expressed as episodes per 1,000 central line days. Categorical variables were compared using a Chi-square test; continuous variables were described by the median, interquartile range (IQR), and were compared using the Student $t$ test. All tests were two-tailed and $p \leq 0.05$ was considered statistically significant.

\section{Results}

During the study period, a total number of patients admitted in LCICU were 1,336 (648 in 2017 and 688 in 2018) and a total of 995 central lines were inserted for various indications. Baseline demographic and clinical data of patients are given in Table 1. A total of 57 patients were meeting the CLABSI criteria among 7,324 central line catheter days of surveillance (Table 2). Before implementation of the insertion and maintenance bundle, the rate of CLABSI was $11.78 / 1,000$ catheter days in 2017 and after implementation of the bundle in 2018 the rate observed was 3.99 per 1,000 catheter days (Figs 1 and 2). The device utilization ratio was 0.73 and 0.77 in years 2017 and 2018, respectively. After full implementation of the CLABSI bundle, there was a $66.1 \%$ annual reduction in the CLABSI rate, which was statistically significant ( $p \leq 0.001)$.

Out of a total 57 patients included in study, 46 (80.7\%) were male. Median (inter quartile range) age of patients was 53 (43-62).

The mean duration from placement of central lines to occurrence of CLABSI was 8.3 days. Median hospital stay was 17 (12-23) and 22 (16-32) days, while the median ICU stay was 14 (9.7-19) and 18 (13-31) days, in years 2017 and 2018, respectively.

The major diagnosis in study subjects was CLD 37 (65\%) followed by ACLF 9 (16\%), ALF 4 (7\%), pancreatitis 3 (5\%), dengue hepatitis $1(2 \%)$, functional metastatic pancreatic neuroendocrine tumor $1(2 \%)$, hepatic venous outflow tract obstruction $1(2 \%)$, and chronic subdural hemorrhage with CLD 1 (2\%). The major comorbidities observed were acute kidney injury 49 (86\%) followed by hepatic encephalopathy 43 (75\%), diabetes mellitus $16(28 \%)$, hypertension 6 (11\%), and chronic kidney disease 4 (7\%).

Gram-negative bacilli (GNB) were the most common pathogens in our study $(49,86 \%)$ followed by gram-positive cocci (GPC) $(7,12.3 \%)$ and non-albicans Candida species (1, 1.8\%). Out of seven $(12.3 \%)$ GPC, we isolated four (7\%) coagulase-negative Staphylococci, one (1.7\%) Staphylococcus aureus (1.8\%), and two (3.5\%) Enterococci faecium (Fig. 3 and Table 3).

All five Staphylococcus species were methicillin-resistant and sensitive to vancomycin, teicoplanin, and linezolid. In addition to these, S. aureus was also sensitive to sulfamethoxazoletrimethoprim and S. capitis was also sensitive to tetracycline and sulfamethoxazole-trimethoprim. Out of two E. faecium, one was vancomycin-resistant Enterococci (VRE) and sensitive to linezolid, while another one was sensitive to vancomycin, teicoplanin, and linezolid.

One $(1.7 \%)$ Candida tropicalis was also isolated, which was sensitive to fluconazole and echinocandins.

Among GNB, we isolated 19 (33.3\%) Pseudomonas aeruginosa, 11 (19.3\%) Klebsiella pneumoniae, 7 (12.3\%) Acinetobacter baumannii, 
Table 1: Demographic and clinical baseline data for the patients

\begin{tabular}{|c|c|c|c|c|}
\hline & $2017(n=42)$ & $2018(n=15)$ & \multirow{2}{*}{$\begin{array}{l}\text { Total }(n=57) \\
\text { Total }\end{array}$} & \multirow[b]{2}{*}{$p$ value } \\
\hline & \multicolumn{2}{|c|}{ Number (\%) } & & \\
\hline \multicolumn{5}{|l|}{ Gender } \\
\hline Male $(n=46)$ & $35(83.3)$ & $11(73.3)$ & $46(80.7)$ & \\
\hline Female $(n=11)$ & $7(16.7)$ & $4(26.7)$ & $11(19.3)$ & \\
\hline Age (median; IQR) & $54(42-62)$ & $52(45-62)$ & $53(43-62)$ & 0.792 \\
\hline \multicolumn{5}{|l|}{ Major diagnosis on ICU admission } \\
\hline Chronic liver disease (CLD) & $27(64)$ & $10(67)$ & $37(65)$ & \\
\hline Acute liver failure (ALF) & $3(7)$ & $1(7)$ & $4(7)$ & \\
\hline Acute on chronic liver failure (ACLF) & $7(17)$ & $2(13)$ & $9(16)$ & \\
\hline Dengue hepatitis & $1(2)$ & 0 & $1(2)$ & \\
\hline Hepatic venous outflow tract obstruction (HVOTO) & 0 & $1(7)$ & $1(2)$ & \\
\hline Pancreatitis & $3(7)$ & 0 & $3(5)$ & \\
\hline Functional metastatic pancreatic neuroendocrine tumor & $1(2)$ & 0 & $1(2)$ & \\
\hline Chronic subdural hemorrhage & 0 & $1(7)$ & $1(2)$ & \\
\hline \multicolumn{5}{|l|}{ Comorbidity } \\
\hline Diabetes mellitus & $9(21)$ & $7(47)$ & $16(28)$ & 0.094 \\
\hline Hypertension & $2(5)$ & $4(27)$ & $6(11)$ & 0.036 \\
\hline Acute kidney injury & $36(86)$ & $13(87)$ & $49(86)$ & 1.00 \\
\hline Chronic kidney disease & $3(7)$ & $1(7)$ & $4(7)$ & 1.00 \\
\hline Hepatic encephalopathy & $34(81)$ & $9(60)$ & $43(75)$ & 0.161 \\
\hline \multicolumn{5}{|l|}{ Symptoms } \\
\hline Fever during IWP & $10(24)$ & $3(20)$ & $13(23)$ & 1.00 \\
\hline \multicolumn{5}{|l|}{ Clinical data (median; IQR) } \\
\hline TLC & $7.6(6.1-12.1)$ & $10(5.2-14.3)$ & $8.5(6.0-13.1)$ & 0.568 \\
\hline $\mathrm{HB}$ & $8.7(7.5-10.3)$ & $8.7(8-10.2)$ & $8.7(7.6-10.3)$ & 0.913 \\
\hline Creatinine & $1.2(0.8-2.5)$ & $1.3(0.8-1.7)$ & $1.3(0.8-2.1)$ & 0.978 \\
\hline Total bilirubin & $5.7(2.6-9.9)$ & $4.4(2.4-8)$ & $5.5(2.2-9.8)$ & 0.337 \\
\hline Aspartate aminotransferase & $64(49-153)$ & $58(39-89)$ & $64(48-105)$ & 0.145 \\
\hline Alanine aminotransferase & $32(22-65)$ & $27(21-47)$ & $32(21-55)$ & 0.281 \\
\hline Alkaline phosphatase & $100(68-130)$ & $100(73-136)$ & $100(71-131)$ & 0.935 \\
\hline Gamma-glutamyl transferase & $32(19-54)$ & $59(36-135)$ & $41(21-63)$ & 0.026 \\
\hline Albumin & $3.9(2.5-4.3)$ & $2.5(2.4-3.2)$ & $2.5(2-2.9)$ & 0.167 \\
\hline Globulin & $3.7(3.4-4.0)$ & $3.6(3.1-3.9)$ & $3.5(2.9-4.3)$ & 0.650 \\
\hline INR & $1.7(1.5-2.2)$ & $1.7(1.6-2.1)$ & $1.7(1.5-2.2)$ & 0.731 \\
\hline Procalcitonin & $1(0.25-1.7)$ & $0.7(0.9-1.2)$ & $0.93(0.2-1.3)$ & 0.257 \\
\hline
\end{tabular}

Table 2: Central line-related information and rate of central line-associated bloodstream infection

\begin{tabular}{llll}
\hline & \multicolumn{2}{c}{ Surveillance year } & \\
\cline { 2 - 3 } LCICU & 2017 & 2018 & Total \\
\hline No. of CVC days & 3,566 & 3,758 & 7,324 \\
No. of patient days & 4,891 & 4,883 & 9,774 \\
No. of CLABSI & 42 & 15 & 57 \\
CLABSI rate/1,000 patient days & 8.587201 & 3.071882 & 5.8318 \\
CLABSI rate/1,000 catheter days & 11.78 & 3.99 & 7.78263 \\
Device utilization ratio & 0.729094 & 0.769609 & 0.74933 \\
Duration from placement of CL to occurrence of CLABSI (mean, $95 \% \mathrm{Cl})$ & $8(5-10)$ & $8(5-13)$ & $8.3( \pm 1)$ \\
Total hospital stay (mean, $95 \% \mathrm{Cl})$ & $17(12-23)$ & $22(16-32)$ & $20.3( \pm 2.8)$ \\
Total ICU stay (mean, $95 \% \mathrm{Cl})$ & $14(9.7-19)$ & $18(13-31)$ & $15(10-21)$ \\
\hline
\end{tabular}

7 (12.3\%) Myroides species, 2 (3.5\%) Burkholderia cepacia group, 2 (3.5\%) Stenotrophomonas maltophilia, and 1 (1.7\%) E. coli. Out of 49 isolates of GNB, 40 (81.6\%) were multidrug resistant (MDR) and 9 (18.4\%) were pan-drug resistant. A total of 17 (89.5\%) Pseudomonas aeruginosa, 7 (63.6\%) K. pneumonia, 3 (57.1\%) A. baumannii, and 1 (100\%) E. coli isolates were sensitive to only colistin and resistant to all available antibiotics used for treatment of GNB. Three (42.9\%) out of 7 isolates of $A$. baumannii, 4 (36.4\%) out of 11 isolates of 
K. pneumonia, and 2 (10.5\%) out of 19 isolates of $P$. aeruginosa were pan-drug resistant including colistin. All seven isolates of Myroides species were sensitive to minocyclin. Both isolates of Burkholderia cepacia group were sensitive to ceftazidime, sulfamethoxazole-trimethoprim, levofloxacin, and minocyclin. Stenotrophomonas maltophilia isolates were sensitive to levofloxacin and sulfamethoxazole-trimethoprim.

\section{Discussion}

Our hospital has a 24-bedded LCICU with an approximate 650 admissions per year and a high CVC insertion rate. Considering the high rate of CVC insertion and mortality associated with BSIs, we conducted a study to determine central lines as the source of the BSIs, and to study the CLABSI rate. This study was aimed to evaluate the effectiveness of implementing a central line insertion and a maintenance bundle. To our knowledge, there have been many surveillance studies for CLABSI but no published surveillance studies from the hepatobiliary unit from India. The present study is the first to have CLABSI surveillance in LCICU. All patients included in our study had primary disease of the hepatopancreatobiliary system. This study emphasizes the efforts that were made to reduce the CLABSI rate and the related microbiological profile including antimicrobial resistance.

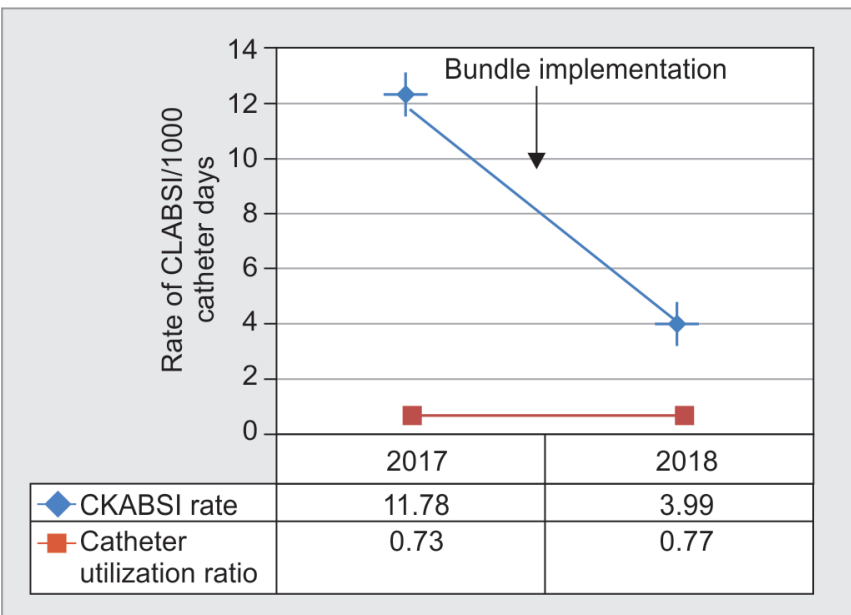

Fig. 1: Impact of implementation of preventive measures
The overall rate of CLABSI in our study was 7.8 per 1,000 catheter days, which was comparable to the study conducted by the International Nosocomial Infection Control Consortium (INICC) in seven Indian cities with reported overall rate of 7.92 per 1,000 catheter days. ${ }^{7}$ The CLABSI rate found in our study was higher than the INICC study conducted from January 2007 through December 2012 in 503 ICUs. ${ }^{8}$ In our study, the incidence was decreased significantly after effective implementation of bundle care. After implementation of bundle, the CLABSI rate was decreased from 11.78 per 1,000 central line days to 3.99 per 1,000 central line days, which was statistically significant (Table 2). We observed $66.1 \%$ annual reduction in the CLABSI rate, which was lower when compared to the study done by Al-Abdullah in Saudi Arabia where they found $95 \%$ reduction. ${ }^{1}$ Differences in CLABSI rates in different studies may be due the differences in degree of implementation and compliance of bundles and differences in microbiological epidemiology. Usually the higher rates of any kind of HAl could be due to the poor nurse-patient ratio, compromised infection-control practices, and poor compliance with the interventional bundle. ${ }^{3}$

Recent studies have demonstrated that consistent application of evidence-based practices can lead to significant, sustained reductions in CLABSI rates. ${ }^{9,10}$ More recently, hospitals using "bundles" have demonstrated great reductions in device-associated HAls and now bundle care has been established to be considered the standard of care. ${ }^{11}$

Gram-negative bacilli were the most common (86\%) isolates in our study. This observation was similar to study by Mansur et al. who reported gram-negative bacteria as most common isolates from CLABSI. ${ }^{12}$ Few studies also shown that gram-positive bacteria are most common agents of CLABSIs. Study by Vinay Khanna et al. reported that $64 \%$ of the pathogens of central line infection were gram-positive and $36 \%$ were gram-negative. ${ }^{13}$ The data reported from developing countries showed that causative microorganisms of CLABSIs were predominantly gram-negative bacteria and this trend of incidence is increasing in recent years. ${ }^{14}$ Studies suggested that with the most preventive measures, reduction in CLABSIs caused by $S$. aureus was more significant than CLABSIs caused by gram-negative bacteria and Candida spp., ${ }^{15}$ but in our study we found reduction in gram-negative bacilli also.

Over the past few years, a concerning trend has been the increasing rate of MDR organisms causing HAls. ${ }^{4}$ In our study, all five Staphylococcus species were methicillin-resistant (four MRCONS

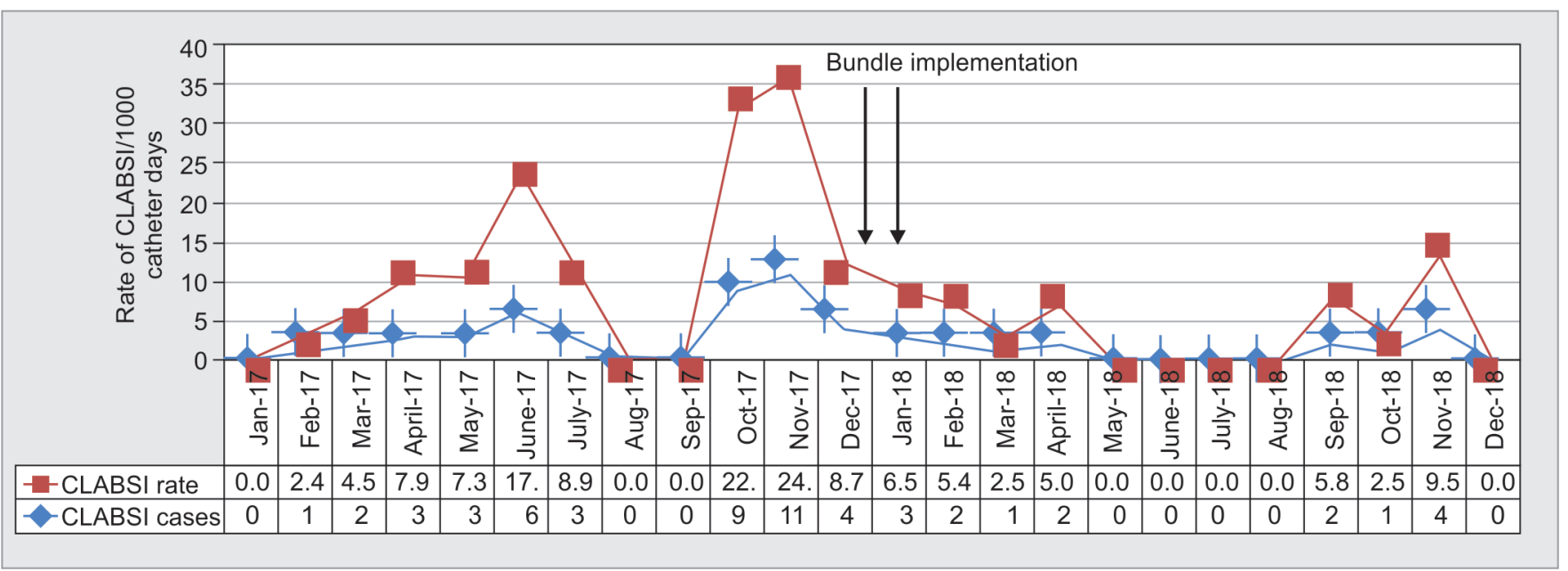

Fig. 2: Liver care intensive care unit central line-associated bloodstream infection rate and incidence 


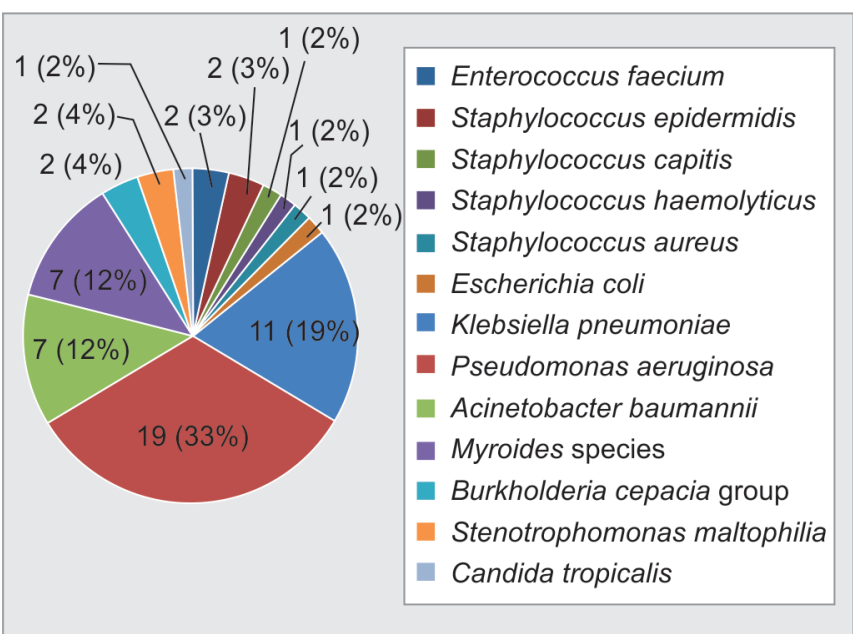

Fig. 3: Microbiological profile of causative microorganisms of central line-associated bloodstream infection

Table 3: Microorganisms causing central line-associated bloodstream infection

\begin{tabular}{|c|c|c|c|}
\hline Organisms & $\begin{array}{l}2017 \\
(n=42)\end{array}$ & $\begin{array}{l}2018 \\
(n=15) \\
\end{array}$ & $\begin{array}{l}\text { Total } \\
(n=57)\end{array}$ \\
\hline Enterococcus faecium & 0 & $2(13.3 \%)$ & $2(3.5 \%)$ \\
\hline Staphylococcus epidermidis & $2(4.7 \%)$ & 0 & $2(3.5 \%)$ \\
\hline Staphylococcus capitis & 0 & $1(6.7 \%)$ & $1(1.7 \%)$ \\
\hline $\begin{array}{l}\text { Staphylococcus } \\
\text { haemolyticus }\end{array}$ & 0 & $1(6.7 \%)$ & $1(1.7 \%)$ \\
\hline Staphylococcus aureus & $1(2.3 \%)$ & 0 & $1(1.7 \%)$ \\
\hline Escherichia coli & 0 & $1(6.7 \%)$ & $1(1.7 \%)$ \\
\hline Klebsiella pneumoniae & $8(19 \%)$ & $3(20 \%)$ & $11(19.3 \%)$ \\
\hline Pseudomonas aeruginosa & $18(43 \%)$ & $1(6.7 \%)$ & $19(33.3 \%)$ \\
\hline Acinetobacter baumannii & $5(12 \%)$ & $2(13.3 \%)$ & $7(12.3 \%)$ \\
\hline Myroides species & $5(12 \%)$ & $2(13.3 \%)$ & $7(12.3 \%)$ \\
\hline Burkholderia cepacia group & $1(2.3 \%)$ & $1(6.7 \%)$ & $2(3.5 \%)$ \\
\hline $\begin{array}{l}\text { Stenotrophomonas } \\
\text { maltophilia }\end{array}$ & $2(4.7 \%)$ & 0 & $2(3.5 \%)$ \\
\hline Candida tropicalis & 0 & $1(6.7 \%)$ & $1(1.7 \%)$ \\
\hline
\end{tabular}

and one MRSA). In study of Sunita et al., out of five CONS, four were MRCONS (80\%), and out of four S. aureus, three were MRSA (75\%). ${ }^{4}$ Out of two E. faecium one was VRE. All the isolated FNB were MDR strains in our study. Hospital-acquired strains are usually MDR as a result of selection pressure of antibiotic use. About 17 (89.5\%) strains of P. aeruginosa, $7(63.6 \%)$ strains of $K$. pneumonia, $3(57.1 \%)$ strains of $A$. baumannii, and $1(100 \%)$ strain of $E$. coli isolate were sensitive to only colistin. Three (42.9\%) out of 7 isolates of $A$. baumannii, 4 (36.4\%) out of 11 isolates of K. pneumonia, and 2 (10.5\%) out of 19 isolates of $P$. aeruginosa were pan-drug resistant. The antimicrobial resistance rate in GNB was higher in our study as compared to other previous studies. ${ }^{4}$ With the increasing resistance, the shortage of newer drug's availability and increasing use of higher antibiotics like carbapenems and last-resort drugs such as colistin as the primary line of treatment in ICUs could lead to the condition of further increase in antibiotic resistance or pan-drug-resistant isolates.

This study had some limitations like our sample size was limited to a single medical center; we did not analyze the compliance rate of our interventional bundle and checklist. Despite these limitations, we provide valuable information on the incidence, etiology, and usefulness of bundle care for prevention of CLABSI in critically ill patients.

Routine surveillance for detection of infection and appropriate preventive measures implementation should be carried out to provide quality of care to patients. Knowledge of the microbiological and antimicrobial susceptibility profile is essential for timely initiation of most appropriate anti-infective therapy.

\section{Conclusion}

Our study was the first to discuss the CLABSI rate in LCICU. The bundle for the prevention of HAls are the standard of care and we found significant reduction in the CLABSI rate after implementation of the bundle. Gram-negative bacteria were the most common pathogen in our study and antimicrobial resistance was very high, which suggest hospital environment as a source of infection.

\section{References}

1. Al-Abdullah N. Epidemiology of central line-associated bloodstream infection (CLABSI) among patients in the intensive care units (ICUs) at a teaching hospital in Saudi Arabia from year 2011-2016. J Intensive Crit Care 2018;4(1):2.

2. Deepti, Sinha S, Sharma SK, Aggarwal P, Biswas A, Sood S, et al. Central Venous Catheter Related Bloodstream Infections in Medical Intensive Care Unit Patients in a Tertiary Referral Centre. Indian J Chest Dis Allied Sci 2014;56(2):85-91.

3. Lin K-Y, Cheng A, Chang Y-C, Hung M-C, Wang J-T, Sheng W-H, et al. Central line-associated bloodstream infections among criticallyill patients in the era of bundle care. J Microbiol Immunol Infect 2017;50(3):339-348. DOI: 10.1016/j.jmii.2015.07.001.

4. Bhatawadekar SM, Arunima, Lahiri KK. Bacterial profile of central line associated blood stream infections in I.C.U patients from tertiary care hospital. IP Int J Med Microbiol Trop Dis 2018;4(1):31-35. DOI: 10.18231/2455-6807.2018.0007.

5. National Healthcare Safety Network, Centers for Disease Control and Prevention. The National Healthcare Safety Network (NHSN) manual: patient safety component protocol. 2018.

6. Rosenthal VD, Al-Abdely HM, El-Kholy AA, AIKhawaja SAA, Leblebicioglu $\mathrm{H}$, Mehta $\mathrm{Y}$, et al. International nosocomial infection control consortium report, data summary of 50 countries for 2010-2015: device-associated module. Am J Infect Control 2016;44(12):1495-1504. DOI: 10.1016/j.ajic.2016.08.007.

7. Mehta A, Rosenthal VD, Mehta Y, Chakravarthy M, Todi SK, Sen N, et al. Device-associated nosocomial infection rates in intensive care units of seven Indian cities. Findings of the international nosocomial infection control consortium (INICC). J Hosp Infect 2007;67(2):168-174. DOI: 10.1016/j.jhin.2007.07.008.

8. Rosenthal VD, Maki DG, Mehta Y, Leblebicioglu H, Memish ZA, Al-Mousa $\mathrm{HH}$, et al. International Nosocomial Infection Control Consortium (INICC) report, data summary of 43 countries for 2007-2012. Device-associated module. Am J Infect Control 2014;42(9): 942-956. DOI: 10.1016/j.ajic.2014.05.029.

9. Apisarnthanarak A, Thongphubeth K, Yuekyen C, Warren DK, Fraser VJ. Effectiveness of a catheter-associated bloodstream infection bundle in a Thai tertiary care center: A 3-year study. Am J Infect Control 2010;38(6):449-455. DOI: 10.1016/j.ajic.2009.08.017.

10. Furuya EY, Dick A, Perencevich EN, Pogorzelska M, Goldmann D, Stone PW. Central line bundle implementation in US intensive care units and impact on bloodstream infections. PLoS One. 2011;6(1):e15452. DOI: 10.1371/journal.pone.0015452.

11. Institute for Healthcare Improvement. Implement the IHI Central Line Bundle. Last modified Aug 2, 2011. Accessed Oct 16, 2013.

12. Mansur FJ, Barai L, Karim MM, Haq JA, Fatema K, Faruq MO. Intravascular catheter related infections and antimicrobial susceptibility pattern 
of isolated bacteria in a tertiary care hospital of Bangladesh. Indian J Med Microbiol 2014;32(1):68-71. DOI: 10.4103/0255-0857. 124321.

13. Khanna V, Mukhopadhayay C, Vandana KE, Verma M, Dabke P. Evaluation of central venous catheter associated blood stream infections: A microbiological observational study. J Pathog 2013;2013:936864. DOI: 10.1155/2013/936864.
14. Marcos M, Soriano A, Inurrieta A, Martinez JA, Romero A, Cobos N, et al. Changing epidemiology of central venous catheter-related bloodstream infections: increasing prevalence of Gram-negative pathogens. J Antimicrob Chemother 2011;66(9):2119e25. DOI: 10.1093/jac/dkr231.

15. Centres for Disease Control and Prevention. Vital signs: central lineassociated blood stream infectionseUnited States 2001, 2008, and 2009. MMWR Morb Mortal Wkly Rep 2011;60(8):243e8. 